\title{
Turbulent and Stable/Unstable Laminar Burning Velocity Measurements from Outwardly Propagating Spherical Hydrogen-Air Flames at Elevated Pressures"
}

\author{
Andrew SMALLBONE ${ }^{* *}$ Kousaku TSUNEYOSHI $^{* *}$ and Toshiaki KITAGAWA ${ }^{* *}$ \\ ${ }^{*}$ Department of Mechanical Engineering Science, Kyushu University, \\ 744 Motooka, Nishi-ku, Fukuoka, Japan \\ E-mail: andrew@mech.kyushu-u.ac.jp
}

\begin{abstract}
The laminar burning velocity of pre-mixed hydrogen-air mixtures was measured in a fan stirred combustion bomb. Unstretched laminar burning velocities and Markstein lengths were obtained at $0.10 \mathrm{MPa}$ for equivalence ratios of $0.4,0.6,0.8$ and 1.0 using high speed flame imaging. The difficulties which arose whilst obtaining similar measurements at $0.25 \mathrm{MPa}$ and $0.50 \mathrm{MPa}$ are outlined. The turbulent burning velocity was measured at equivalence ratios of 0.4 and 0.8 from explosions carried out at $0.10 \mathrm{MPa}$ with turbulence intensities of 0.8 and $1.6 \mathrm{~m} / \mathrm{s}$. Higher turbulent burning velocity ratios were observed for mixtures which yielded lower Markstein lengths in the laminar combustion experiments.
\end{abstract}

Key words: Hydrogen, Combustion, Laminar Burning Velocity, Turbulent Burning Velocity, Markstein Length, Flame Instability

\section{Introduction}

A hydrogen fuelled SI engine would be advantageous due to the associated reduction in $\mathrm{CO}_{2}$ emissions and improved energy security as fossil fuels are depleted. Whilst practical hydrogen powered fuel cells are in development, hydrogen (either bi-fuel or fully) fuelled SI engines could provide an immediate solution based on current and well established technology ${ }^{(1)}$. Hydrogen fuelled SI engines are operated in lean combustion mode and without significant intake throttling, with the associated efficiency benefits and reduced NOx exhaust emissions.

Fundamental combustion data such as the laminar burning velocity have formed the basis of the development of reliable predictive turbulent combustion models/correlations adopted by the automotive industry ${ }^{(2),(3)}$. However, presently many such models/correlations cannot adequately reproduce the influence of thermo-diffusion on turbulent flames ${ }^{(4)}$. Unstable laminar flames have been observed to be more unstable with a more "fine" and "convoluted" structure (5) and consequently a larger flame front. Turbulent combustion rates were shown to be further enhanced at those conditions where as laminar flames, greater instability was observed ${ }^{(4)}$. These aspects are often characterised by adopting a Markstein length from laminar flame studies, which has been shown to be influenced significantly by the fuel type, mixture strength and pressure ${ }^{(6)-(12)}$.

It has been reported that hydrogen-air mixtures are more sensitive to these aspects than gasoline fuels ${ }^{(6),(10)}$, which has proven important since the equivalence ratio is also varied to control the load in hydrogen fuelled SI engines ${ }^{(1)}$. Hence, the influence of instability and thermo-diffusion must be considered for any model or correlation adopted to predict

*Received 28 Dec., 2005 (No. 05-0008) [DOI: 10.1299/jtst.1.31] 
hydrogen fuelled combustion.

This study outlines the experimental techniques adopted at Kyushu University for the study of spherically propagating turbulent and laminar flames with methane/propane-air. These techniques are used to analyse explosions of hydrogen-air mixtures obtained at 0.10 , 0.25 and $0.50 \mathrm{MPa}$. Measurements of the unstretched laminar burning velocity and Markstein length carried out at $0.10 \mathrm{MPa}$ are presented and compared with other data available in the literature. The limitations of adopting these techniques for those explosions carried out at 0.25 and $0.50 \mathrm{MPa}$ are highlighted. Data from turbulent explosions carried out with turbulence intensities (or turbulent r.m.s. velocities) of 0.8 and $1.6 \mathrm{~m} / \mathrm{s}$ are presented and the influences of local stretch rates and flame instability on turbulent flame propagation are addressed.

\section{Experimental set-up}

Experiments were carried out using the constant volume bomb shown schematically in Fig. 1. The combustion vessel at Kyushu University is comprised of three orthogonal intersecting cylinders each of $265 \mathrm{~mm}$ diameter with a corresponding total volume of approximately $35,000 \mathrm{~cm}^{3}$ or equivalent to that of a sphere of $406 \mathrm{~mm}$ diameter.

The vessel is made from steel and capable of withstanding initial pressures of up to 1.0MPa. The opposite ends of the horizontal intersecting cylinder have been fitted with a pair of quartz glass windows of $200 \mathrm{~mm}$ diameter and $110 \mathrm{~mm}$ thickness. These windows were used to view a cylindrical corridor, of diameter of $160 \mathrm{~mm}$, where photographic techniques and laser anemometry were adopted for visualising flame development and to determine the turbulence scales respectively.

A schematic diagram of the experimental apparatus is presented in Fig. 2. Two identical fans were rotated using two motors mounted on the top and bottom of the combustion vessel, these fans comprised of four blades and were fitted oppositely within the vertically arranged intersecting cylinder. The voltage to each of the motors was controlled using a variable resistor. A shaft encoder and Yokogawa FC-863 frequency counter were employed to monitor the fan rotational speed. The amount of turbulence within the vessel was controlled by varying the fan rotational speed.

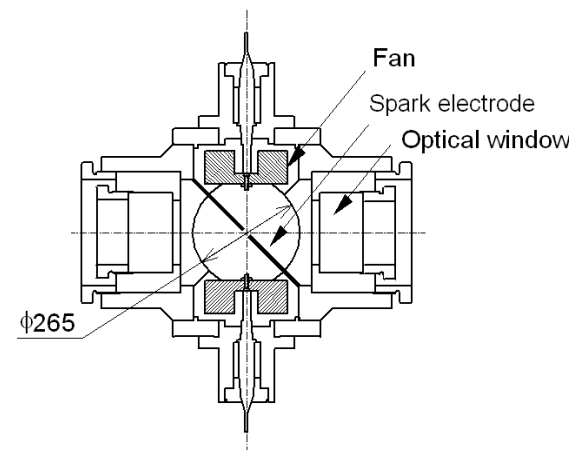

Fig. 1 Schematic diagram of the combustion vessel $^{(13)}$

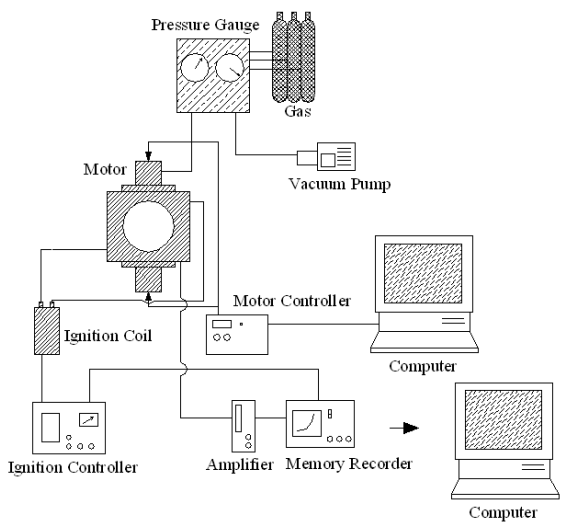

Fig. 2 Schematic of experimental setup ${ }^{(13)}$

\subsection{Turbulence measurements}

A correlation between fan rotational speed, turbulence intensity and integral length scale was developed using data obtained from Particle Image Velocimetry (PIV) measurements carried out by KANOMAX Japan Inc.

Laser light from a YAG Newwave Solo laser at $50 \mathrm{~mJ} /$ pluse was pulsed twice (with a delay of $15 \mu \mathrm{s}$ ) at $3.7 \mathrm{~Hz}$ into an area of $44 \times 34 \mathrm{~mm}$ within the central region of the 
combustion vessel via a LLS-YAG lens with focal length of $500 \mathrm{~mm}$. The laser sheet thickness was approximately $250 \mu \mathrm{m}$. Smoke from incense sticks was used as particle tracing seed and a TSI PIVCAM 13-8 camera was employed to image the turbulent flow. One hundred image pairs were stored per condition at a resolution of 1280 by 1024 pixels (or $\sim 0.033 \mathrm{~mm} / \mathrm{pixel}$ ) with a dynamic range of $12 \mathrm{bit}$. Each image was separated into squares of 32 by 32 pixels (with $50 \%$ overlap) and corresponding velocity vector components were resolved, Fig. 3. These vectors were adopted to compute mean bulk velocity vectors and the deviation used to determine r.m.s velocity components (or turbulence intensity) based on turbulence, $u^{\prime}$ and $v^{\prime(14)}$.

Presented in Fig. 4 are the corresponding measurements of turbulence intensity at 0.10, 0.25 and $0.50 \mathrm{MPa}$ for fan speeds of 1800,2700 and $3600 \mathrm{rpm}$. Increasing mixture pressures did not influence the noted turbulence intensity, hence a linear relationship between fan speed, $N$ and mean turbulence intensity, $\mathrm{u}^{\prime}$ is presented.

In the centre of the combustion vessel, the turbulence was considered homogeneous with no regular bulk motions. The same data was adopted to obtain an integral length scale of turbulence based on the spatial cross-correlation technique. This technique yielded a longitudinal integral length scale, $L_{f}$ of $1.2 \mathrm{~mm}$, which similarly proved independent of pressure.

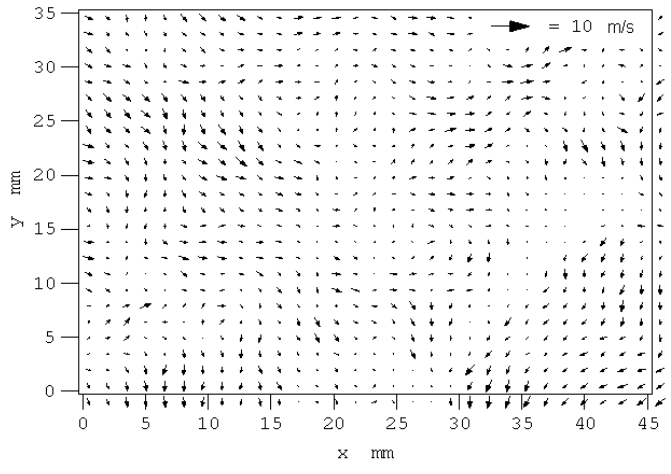

Fig. 3 Vector map of turbulent flow with a fan rotational speed of $1800 \mathrm{rpm}$ at $0.1 \mathrm{MPa}^{(13)}$

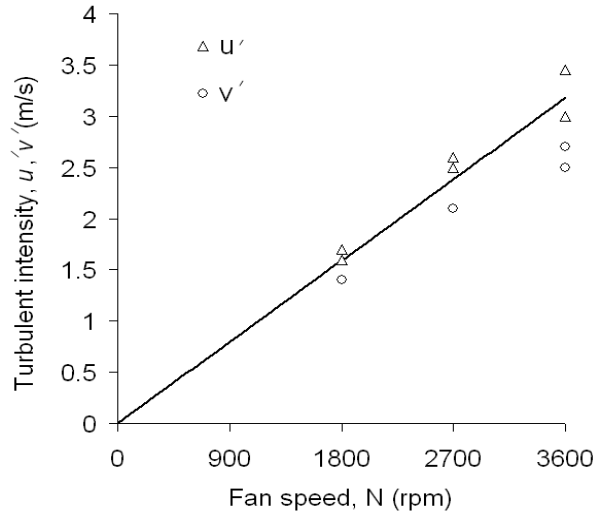

Fig. 4 Fan rotational speed versus turbulence intensity, $u^{\prime}$

\subsection{Mixture preparation}

In preparation for an explosion, the vessel was brought to a vacuum. Dry air and hydrogen (purity of 99.97\%) supplied by Sumito Seika Chemicals Co. Ltd. was supplied to the vessel in proportions based on its partial pressure. Explosions were carried out for various initial pressures, $P_{i}$. This pressure was monitored using a Druck DPI260 pressure transducer and Druck pressure indicator display unit. Throughout this process, the fans were operated to ensure proper mixing - however if a laminar explosion was planned, the fans were switched off for at least two minutes prior to ignition, to ensure a quiescent mixture. After the explosion, the exhaust valve was opened and a vacuum pump was operated to vent any undesired residual exhaust gases. The combustion vessel door was opened and all water deposits (from the exhaust gas) were wiped dry by hand, the insides of the vessel were then cooled using an airgun.

The spark electrode comprised two electrodes of $1.8 \mathrm{~mm}$ diameter mounted on opposite sides of the combustion chamber. The clearance between electrodes at the centre of the chamber was approximately $3 \mathrm{~mm}$ for all test conditions. A spark was induced by charging a $32 \mu \mathrm{F}$ capacitor at $300 \mathrm{~V}$, and when required this energy was discharged to the ignition coil, the total energy supplied to the spark was approximated as $1.4 \mathrm{~J}$ in this study.

Laminar explosions were carried out with initial pressures, $P_{i}$ of $0.10,0.25$ and 
$0.50 \mathrm{MPa}$ and equivalence ratios of $0.4,0.6,0.8$ and 1.0. Turbulent explosions were conducted with an initial pressure of $0.10 \mathrm{MPa}$ with equivalence ratios of 0.4 and 0.8 , for turbulent intensities of 0.8 and $1.6 \mathrm{~m} / \mathrm{s}$.

\subsection{Schlieren/imaging photography}

Equipment and technique: The optical technique to determine burning velocities was preferred, as corresponding pressure/temperature increases were considered relatively small (with a $0.1 \mathrm{MPa}$ initial pressure and maximum observed flame diameter of $160 \mathrm{~mm}$, the total pressure rise was approximately $0.05 \mathrm{MPa}$ ). Hence, burning velocities were measured at approximately constant pressure and temperature. Furthermore, through the visualisation of flames, the influence of the onset of cellularity and instability could be assessed. Schlieren photography was preferred to natural light photography since it had the advantage of providing a more defined image of the flame surface.

The experimental arrangement for schlieren imaging is presented in Fig. 5. A 500W Ushio Optical Module X Xenon lamp was employed as a light source, the light beam passed through a $4 \mathrm{~mm}$ pinhole and a series of ND (Neutral Density) filters (to adjust the light to the desired intensity). A Sigma Kouki convex lens with $190 \mathrm{~mm}$ diameter and $2.5 \mathrm{~m}$ focal length was employed to from a parallel beam which passed through the combustion chamber. Due to limited available space in the lab, a $250 \mathrm{~mm}$ convex mirror (made by Mizojiri Optical Co. Ltd.) with a focal length of $2.5 \mathrm{~m}$ was used to focus the beam onto a user variable sized "pinhole". The divergent beam then passed into a Phantom high speed digital camera which was nominally operated at $1000 \mathrm{f} / \mathrm{s}$ with $512 \times 512$ pixel resolution (spatial resolution $\sim 0.3 \mathrm{~mm} / \mathrm{pixel})$. Combustion was very fast $(>4.0 \mathrm{~m} / \mathrm{s})$ for turbulent explosions at an equivalence ratio of greater than 0.8 , hence the framing rate was increased.

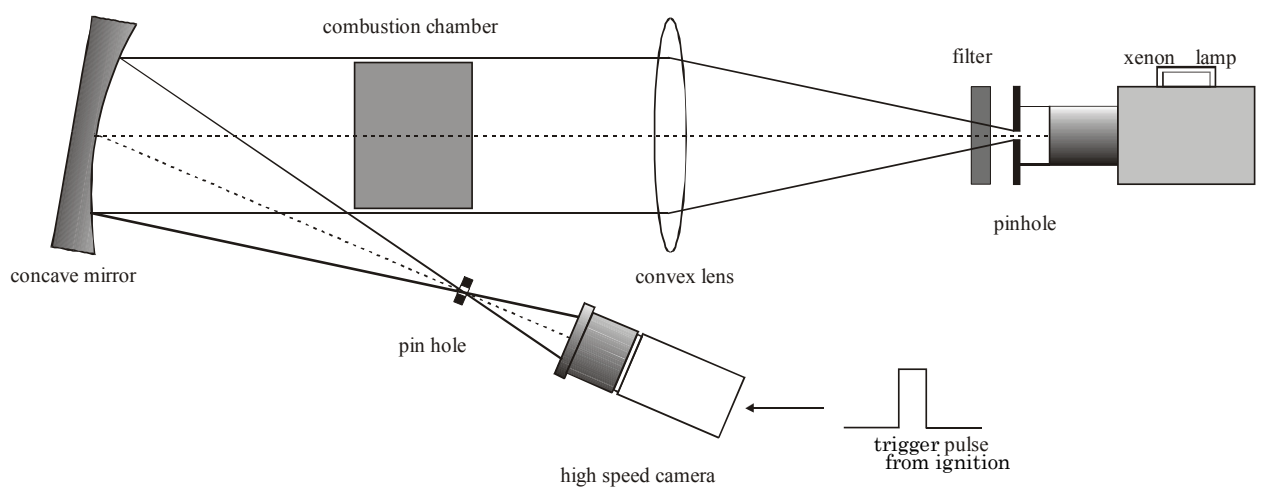

Fig. 5 Schematic diagram of the schlieren experiments ${ }^{(13)}$

Processing: Raw flame images were converted to a TIFF image using the Phantom software. Images were converted from the raw greyscale image into a binary black and white image, using the Adobe Photoshop software. A program developed at Kyushu University was used to process the images to determine an enflamed area, flame perimeter and equivalent circular schlieren flame radius, $r_{s c h}$.

\section{Laminar flames}

Spherically expanding premixed flames have been reported to be influenced by the variable flame stretch rate ${ }^{(6)-(12)}$ and hence flame stretch rate must be considered. At low flame radii, the flame stretch rates were high and decreased with flame radii.

Unstretched laminar burning velocities were determined using the following approach. An observed mean schlieren flame radius, $r_{s c h}$ versus time of a stoichiometric methane-air explosion at $0.10 \mathrm{MPa}$ is presented in Fig. 6(a). This radius was adopted to determine a stretched laminar burning velocity, $u_{n}{ }^{(6),(7),(8)}$ by considering the influence of hot burned gas 
expansion.

$$
u_{n}=\frac{\rho_{b}}{\rho_{u}} S_{n}=\frac{\rho_{b}}{\rho_{u}} \frac{\mathrm{d} r_{s c h}}{\mathrm{~d} t}
$$

where, $\rho_{u}$ and $\rho_{b}$ are the densities of the unburned mixture and burned gas respectively, $S_{n}$ corresponds to the flame propagation speed and $t$ is time.

The flame stretch rate, $\alpha$ of a spherically propagating flame was determined from the flame front area $A$, or the flame radius, $r_{s c h}$ according to Eq. (2) ${ }^{(6),(7),(8)}$.

$$
\alpha=\frac{1}{A} \frac{\mathrm{d} A}{\mathrm{~d} t}=\frac{2}{r_{s c h}} S_{n}=\frac{2}{r_{s c h}} \frac{\mathrm{d} r_{s c h}}{\mathrm{~d} t}
$$

The corresponding stretched laminar burning velocity, $u_{n}$ and flame stretch rate, $\alpha$ for a stoichiometric methane-air flame at $0.10 \mathrm{MPa}$ is presented in Fig. 6(b). Once the flame was considered free from the influence of the spark ( $r>6 \mathrm{~mm}$ and discussed in more detail later) ${ }^{(14)}$, this relationship was approximated as linear. Hence, by extrapolation of data to a zero flame stretch rate (intercept value of the vertical axis), an unstretched laminar burning velocity, $u_{l}$ was obtained. The effect of variable flame stretch rate on a flame has often been characterised by the Markstein length, $L^{(6),(7),(8)}$ which was determined by calculating the gradient of the line. These relationships can be summarised by Eq. (3) ${ }^{(6),(7),(8)}$.

$$
u_{1}-u_{n}=L \cdot \alpha
$$

Furthermore, Eq. (3) can be non-dimensionalised by normalising by the unstretched laminar burning velocity and the laminar flame thickness, $\delta_{l}$ :

$$
\frac{u_{l}-u_{n}}{u_{l}}=K \cdot M a
$$

where $K$ is the Karlovitz number and $M a$ is the Markstein number ${ }^{(6),(7),(8)}$.

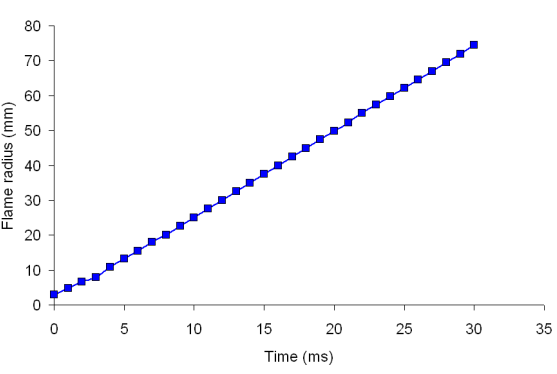

(a) Flame radius versus time

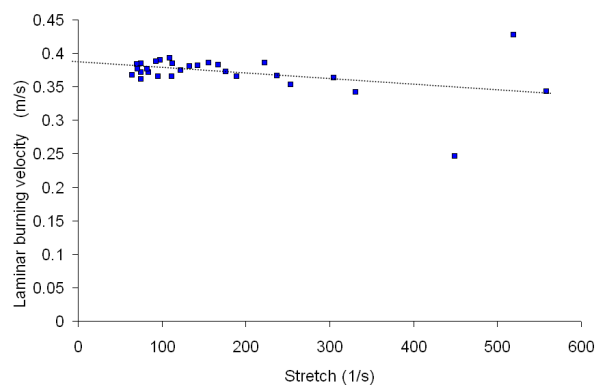

(b) Laminar burning velocity versus stretch rate

Fig. 6 Data from a methane-air flame with an equivalence ratio of 1.0 at 0.1MPa.

\subsection{Hydrogen-air flames}

Corresponding laminar burning velocities, $u_{n}$ versus flame stretch rate, $\alpha$ for explosions carried out with hydrogen-air mixtures are presented in Fig. 7. The variation in the laminar burning velocity between the three explosions carried out at each test condition was considered small. Hence those data points from all three explosions are presented to increase the number of available data points.

As the spherical flames propagated outwardly, the flame stretch rate reduced. At all conditions, increased laminar burning velocities were eventually noted with reduced stretch rates. However, at high flame stretch rates, increased laminar burning velocities were not always observed with reducing flame stretch rates. This was most evident at an equivalence ratio of 0.4 with flame stretch rates greater than $750 \mathrm{~s}^{-1}$. In such a regime, reduced laminar burning velocities were observed with increasing pressure. At all other equivalence ratios, the laminar burning velocity was smallest at greater stretch rates. However in all such cases, increasing pressure resulted in increasing laminar burning velocity.

The sudden increase in laminar flame speeds was expected to be associated with the 
transition from stable flame into unstable (cellular) flame propagation, this is marked on Fig. 7(b). It was expected that all explosions presented in Fig. 7 were eventually influenced by unstable flame development.

\subsection{Unstable flame propagation}

To highlight the sensitivity of hydrogen-air flames to unstable flame development, corresponding images of all laminar explosions with a flame stretch rate of $750 \mathrm{~s}^{-1}$ are presented in Fig. 8. The laminar burning velocity of leaner mixtures was typically slower, hence the adopted flame stretch rate occurred at a smaller flame radii. At this flame stretch rate, the flame front appeared smoother and less convoluted for those explosions carried out at lower pressures.

The relationship between stretch rate, Markstein number and onset of unstable flame development has been comprehensively discussed by Bradley ${ }^{(9)}$. It has been shown that all flames are influenced by the underlying Darrieus-Landau instability; however local thermodiffusive effects can either reduce or reinforce the instability. In spherically propagating flames, cellular (unstable) flames are observed once a particular stretch rate is exceeded. This stretch rate has been shown to be dependant on the fuel, equivalence ratio and pressure.

Thermodiffusive properties of flames are often generalised by adopting a Lewis number, a ratio of the rate of thermal and molecular diffusion ${ }^{(3),(8)}$

$$
L e=\frac{\lambda}{\rho c_{p} D}
$$

where $\lambda$ is the thermal conductivity, $\rho$ is the gas density, $c_{p}$ is the constant pressure specific heat of the mixture and $D$ is the diffusion coefficient of the deficient reactant. For mixtures where thermal diffusion has been shown as most dominant and at higher rates of stretch, the underlying instability has been shown to be partially suppressed and a stable laminar flame region observed. With methane-air flames with an equivalence ratio of 0.8 , this behaviour would be expected as the Lewis number is $L e=1.01$. However, corresponding hydrogen-air flames were considered more dominated by molecular diffusion with corresponding Lewis number of $L e=0.57$. Hence, the onset of instability was more advanced by thermodiffusive effects. Lean hydrogen-air flames are fundamentally more unstable than methane-air flames.

As noted with similar studies of methane-air and propane-air mixtures (6),(7),(8), hydrogen-air mixtures also proved unstable with increasing pressure. Once the flame front became unstable, it was expected that the increase in surface area contributed to the observed increase in stretched laminar burning velocities with increasing pressures.

\subsection{Laminar burning velocity and Markstein length}

For the reasons outlined above, it follows that the whole range of stretch rates noted in the laminar explosions presented in Fig. 7 were not adopted to determine an unstretched laminar burning velocity and Markstein length. Hence, only measurements obtained within the stable flame regime were selected, the stable regime was defined on the basis of two limits.

The lower limit was set as the point where stretch rate proved too small to continue to suppress the Darrieus-Landau instability. This was characterised by significant visible cellular flame formation and a rapid increase in the laminar burning velocity.

The higher limit was determined by considering flames at small flame radii. Initial flame kernels and small flames have been shown to be influenced by;

1. Spark energy: The spark energy for the initial flame kernel was investigated by Bradley et al. ${ }^{(15)}$, these workers demonstrated that early flame only became independent of initial flame energy once the flame radii were greater than 
approximately $6 \mathrm{~mm}$.

2. Heat transfer: Heat transfer from the flame to the spark electrode may well reduce the temperature of the flame and thus influence burning velocity.

Hence, the criteria of a $6 \mathrm{~mm}$ minimum flame radii was adopted for the maximum stretch rate for determination of an unstretched laminar burning velocity.

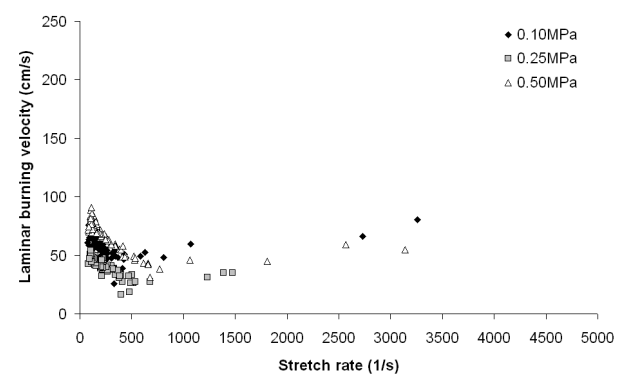

(a) Equivalence ratio of 0.4

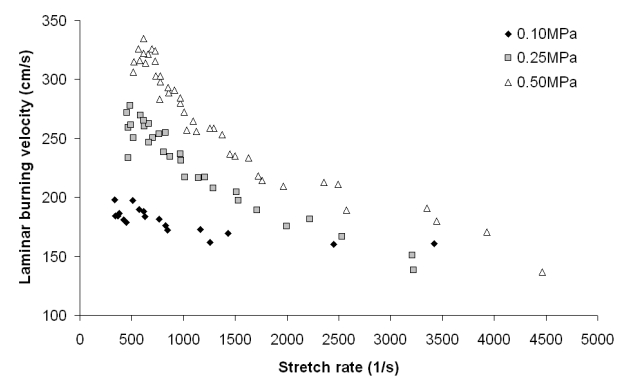

(c) Equivalence ratio of 0.8

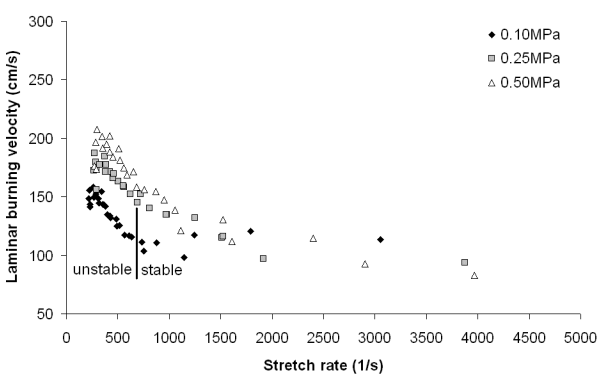

(b) Equivalence ratio of 0.6

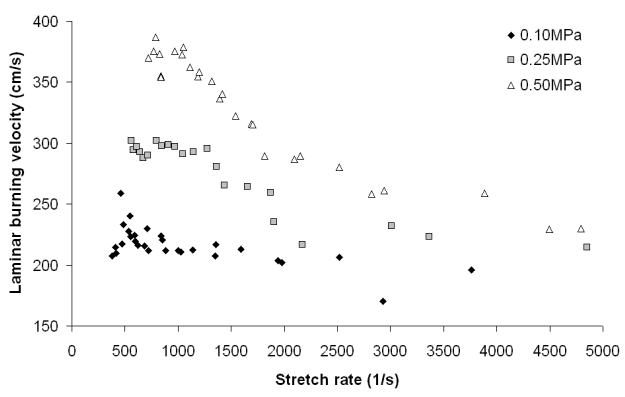

(d) Equivalence ratio of 1.0

Fig. 7 Laminar burning velocity versus stretch rate, $\alpha$ for initial pressures of $0.10,0.25$ and $0.50 \mathrm{MPa}$.

Measurements of unstretched laminar burning velocity and Markstein length at initial pressure of 0.10MPa are presented in Fig. 9(a) and Fig. 9(b) together with some of the corresponding data for hydrogen-air mixtures from alternative studies (10), (11), (12), (16). Generally, leaner mixtures were observed to have lower unstretched laminar burning velocities with increasingly negative Markstein lengths.

The unstretched laminar burning velocities and Markstein lengths obtained in this study compared well with other published measurements, however at lean equivalence ratios, the quantity of available data in the stable flame region proved insufficient. Hence, resulting measurements were considered more prone to error, this may well explain the differences between the measurements presented from this study and those published elsewhere.

Due to the formation of cellular flames with flame radii of less than $6 \mathrm{~mm}$, unstretched laminar burning velocities and Markstein numbers were not determined at 0.25 and $0.50 \mathrm{MPa}$. However from the available data, it was noted that the formation of cellular structures was more rapid for increasing pressure and for leaner mixture strengths. In comparison with similar studies of methane-air and propane-air mixtures ${ }^{(6),(7),(8)}$, this could well be associated with reduced Markstein numbers for these conditions. Measurements also indicated that burning velocities of hydrogen-air flames are severely influenced by unstable flame propagation particularly at elevated pressures. 


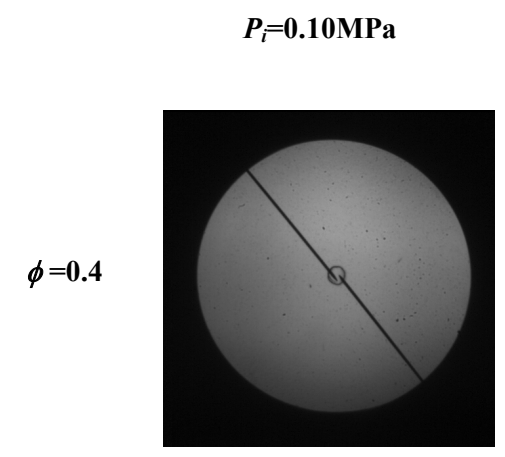

$P_{i}=0.25 \mathrm{MPa}$
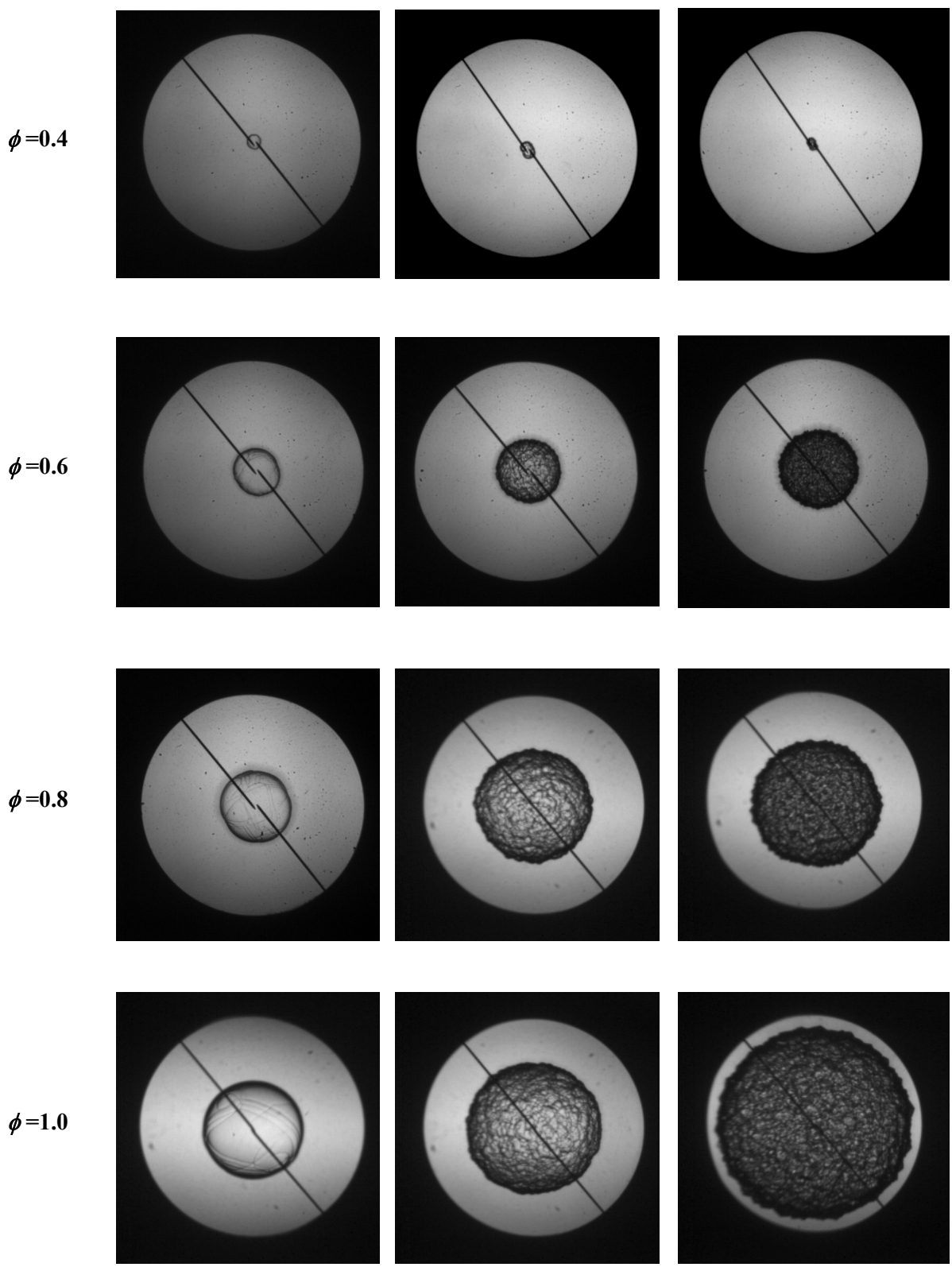

Fig. 8 Images from laminar explosions at a stretch rate of $750 \mathrm{~s}^{-1}$

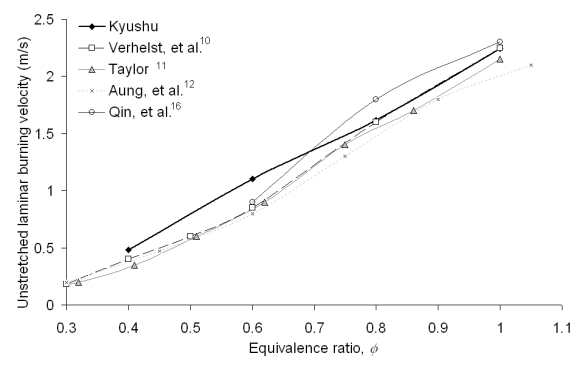

(a) Unstretched laminar burning velocity versus equivalence ratio

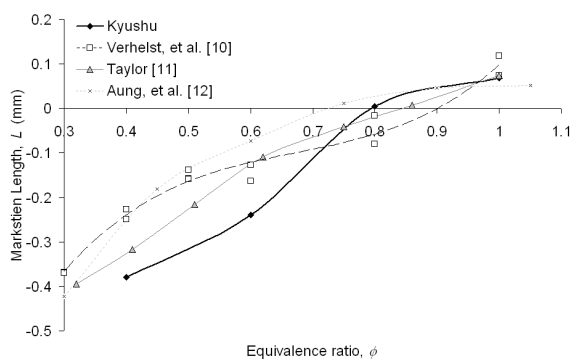

(b) Markstein length versus equivalence ratio

Fig. 9 Measurements for hydrogen-air flames at 0.10MPa 


\section{Turbulent flames}

Turbulent explosions were carried out at $0.10 \mathrm{MPa}$, with turbulence intensities of 0.8 and $1.6 \mathrm{~m} / \mathrm{s}$ and for equivalence ratios of 0.4 and 0.8 . Resulting explosions were considered to be in the wrinkled laminar flame regime. Since turbulent flame propagation in the combustion vessel was also prone to the same processes which can lead to the cycle-to-cycle variations noted in SI engines, at each condition, turbulent explosions were carried out until three similar explosions (on the basis of pressure and flame radii) were observed.

Mean schlieren radii, $r_{s c h}$ were calculated by determining a circle of equivalent area to the observed turbulent flame. Turbulent burning velocities, $u_{t n}$ were obtained by multiplying the flame propagation speed by the density ratio ${ }^{(18)}$.

$$
u_{t n}=\frac{\rho_{b}}{\rho_{u}} \frac{\mathrm{d} r_{s c h}}{\mathrm{~d} t}
$$

Presented in Fig. 10 are the corresponding turbulent burning velocities versus time of the turbulent explosions recorded in this study. Typically for turbulent flames, as the flame developed, it was influenced by a wider spectrum of turbulent scales and the burning velocity increased with time. Similarly, the burning velocity proved greater for the increased turbulence intensity. As noted with measurements of laminar burning velocity, combustion was fastest for greater equivalence ratios.

Turbulent combustion can be characterised by normalising the turbulent burning velocity by the unstretched laminar burning velocity or otherwise known as the "burning velocity ratio". Similarly, the influence of variable turbulence intensities on flame development and turbulent "flame strain" can be normalised by adopting a dimensionless turbulence time scale ${ }^{(2)}$. The dimensionless turbulence time, $t_{k}$ was defined as;

$$
t_{k}=\left(\frac{8}{\pi}\right)^{0.5} \frac{t}{L_{f} / u^{\prime}}
$$

where $t$ is time, $L_{f}$ is the longitudinal integral length scale and $u^{\prime}$ is the turbulence intensity.

Presented in Fig. 11 are data from the turbulent explosions, shown in terms of dimensionless time and burning velocity ratio. With an equivalence ratio of 0.8 , explosions carried out with a turbulence intensity of 0.8 and $1.6 \mathrm{~m} / \mathrm{s}$ were noted to have the same burning velocity ratios for a given dimensionless time. Under laminar conditions, such a flame was observed to have a Markstein length of approximately zero and hence by definition the laminar burning velocity was independent of stretch rate. The turbulent burning velocity of those explosions carried out with an equivalence ratio of 0.4 , were not so similar, however differences were considered too subtle to be above experimental uncertainties.

At $\phi=0.4$, burning velocity ratios were greater than at $\phi=0.8$. This may well have been because the adopted value for unstretched laminar burning velocity was too small for the observed turbulent burning velocity. By adoption of a burning velocity ratio, the unstretched laminar burning velocity was used to represent the "local reaction rate" within a turbulent flow. However it was regarded as inadequate at this condition, hence further consideration of the thermo-diffusive properties of the flame and the Markstein length may well be required.

Recent studies by Kitawaga et al. ${ }^{(8),(19)}$ have discussed the influence of Markstein length on turbulent flames for propane and methane. In this study, an equivalence ratio of 0.4 yielded a negative Markstein length. Since in practice, it would be expected that the flame would be stretched in a positive manor ${ }^{(17)}$, the local reaction rate would be greater than the unstretched laminar burning velocity. An increased laminar burning velocity may well explain the differences between those data presented in Fig. 11. 


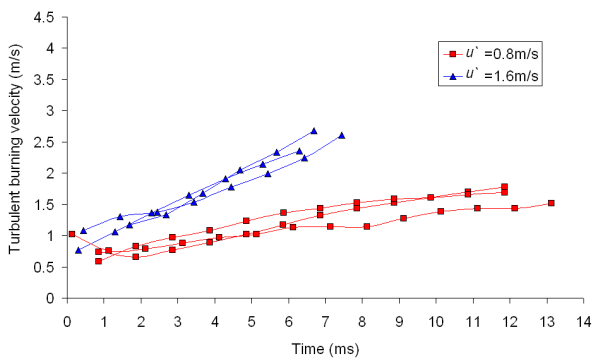

(a) Equivalence ratio of 0.4

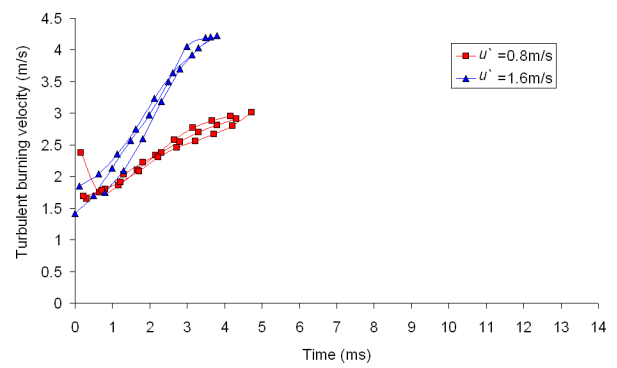

(b) Equivalence ratio of 0.8

Fig. 10 Turbulent burning velocity versus time of explosions carried with turbulence intensities of 0.8 and $1.6 \mathrm{~m} / \mathrm{s}$.

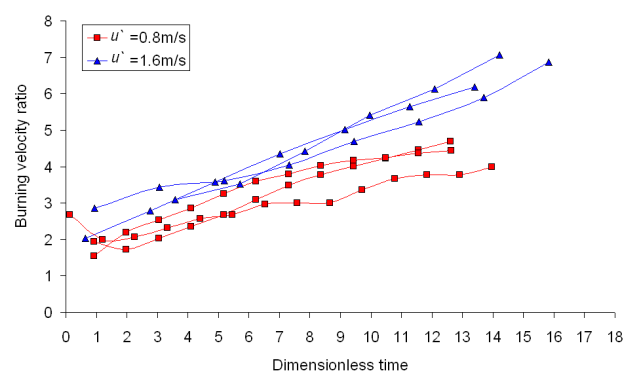

(a) Equivalence ratio of 0.4

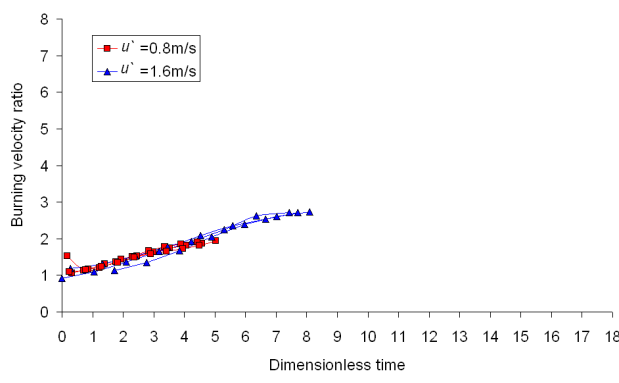

(b) Equivalence ratio of 0.8

Fig. 11 Turbulent to laminar burning velocity ratio versus dimensionless time of explosions carried with turbulence intensities of 0.8 and $1.6 \mathrm{~m} / \mathrm{s}$.

Reliable turbulent burning velocities were obtained for hydrogen-air mixtures using the same techniques as adopted for methane-air experiments. However, even in turbulent flames understanding the influence of Markstein length and thermo-diffusion on the local laminar burning velocity has proven necessary. Thus an improved understanding of turbulent hydrogen combustion must come through simultaneous analysis of laminar flames. However, for hydrogen-air flames it has proven difficult to obtain such measurements reliably at elevated pressures.

\section{Conclusions}

1. A spherical combustion vessel has been developed which can withstand initial pressures of up to 1.0MPa. The turbulent intensity and integral length scale have been measured and can be controlled within the vessel.

2. Reliable laminar burning velocity measurements have been obtained within the stable laminar flame region. The size of this region was limited by the influence of the initial spark kernel and by the onset of flame instability.

3. The unstretched laminar burning velocity and Markstein numbers of hydrogen-air mixtures at $0.10 \mathrm{MPa}$ have been determined. However, due to a low data resolution for the more unstable mixtures, the Markstein numbers were generally lower than those published elsewhere.

4. Those mixtures with a lower Markstein length, were shown to have a greater turbulent burning velocity than expected, based on their unstretched laminar burning velocity. The local stretch rates within the turbulent flame brush were expected to have increased the local burning velocity and hence overall turbulent burning velocity. 


\section{References}

(1) Tang, X. et al. (2002) "Ford P2000 hydrogen engine dynamometer development" SAE Paper 2002-01-0242.

(2) Lipatnikov, A. N. and Chomiak, J. (1997) “A Simple Model of Unsteady Turbulent Flame Propagation" SAE Paper 972993.

(3) Bradley, D. et al. (1992) "Flame stretch rate as a determinate of turbulent burning velocity" Philosophical Transactions of the Royal Society, A, 338: pp359-387.

(4) Lipatnikov, A. and Chomiak J. (2005) "Molecular transport effects on turbulent flame propagation and structure" Progress in Energy and Combustion Science 31:pp1-73.

(5) Kobayashi, H. (2002) "Experimental study of high-pressure turbulent premixed flames" Experimental Thermal and Fluid Science 26:pp375-387.

(6) Bradley, D. et al. (1998) "The measurement of laminar burning velocities and Markstein numbers for iso-octane air and iso-octane - n-heptane-air mixtures at elevated temperatures and pressures in an explosion bomb" Combustion and Flame 155: pp126-144.

(7) Kitagawa, T. et al. (2004) "Effects of pressure on instability and cellularity of propagating spherical laminar flames ( $1^{\text {st }}$ report, cell formation and critical Peclet number)"JSME Journal, 70-698, B, 2649-2656

(8) Kitagawa, T. et al. (2005) "The effects of pressure on turbulent burning velocity of premixed flames and their correlations with Lewis number" The $18^{\text {th }}$ Internal combustion engine symposium (International), Dec. 2005.

(9) Bradley, D. (1999) "Instabilities and flame speeds in large-scale premixed gaseous explosions" Philosophical Transactions of the Royal Society, A, 357:pp3567-3581.

(10) Verhelst, S. et al. (2005) "Laminar and unstable burning velocities and Markstein lengths of hydrogen-air mixtures at engine-like conditions" Proceedings of the Combustion Institute 30: pp209-216.

(11) Taylor, S. C. (1991) "Burning velocity and the influence of stretch" PhD Thesis, Leeds University.

(12) Aung, K. T. et al. (1997) "Flame stretch interactions of laminar premixed hydrogen-air flames at normal temperature and pressure" Combustion and flame, 109: pp1-24.

(13) Harada , K. (2002) "Effects of Pressure on Characteristics of Propagating Premixed Flames (in Japanese) " Masters Thesis, Kyushu University

(14) Hinze, J. O. (1959) "Turbulence: An introduction to its mechanism and theory" McGraw-Hill Book Company, Inc.

(15) Bradley, D. et al. (1996) "Burning velocities, Markstein lengths and flame quenching for spherical Methane-air flames: A computational study” Combustion and Flame 104: pp176-198.

(16) Qin, X. et al. (2000) "Laminar burning velocity of hydrogen-air pre-mixed flames at elevated pressure” Experimental Thermal and Fluid Science 21: pp58-63.

(17) Renou, B. et al. (2000)"Local Scalar Flame Properties of Freely Propagating Premixed Turbulent Flames at Various Lewis Numbers" Combustion and Flame, 123, (2000), 507-521.

(18) Bradley, D. et al. (2003) "Turbulent burning velocity, burned gas distribution, and associated flame surface definition" Combustion and Flame 133 (2003) 415-430.

(19) Kitagawa, T., et al. (2005) "Study on the Effects of Pressure on Turbulent Burning Velocity of Outwardly Propagating Methane-Air Flame with Reynolds and Markstein Numbers", Transactions of Society of Automotive Engineers of Japan, Vol.36, No.6, 13-18. 